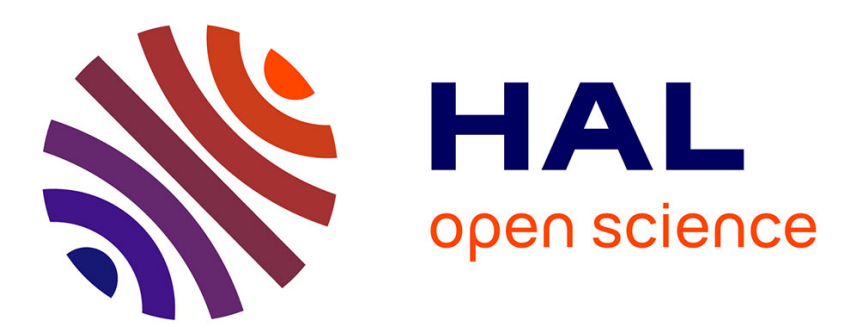

\title{
An Overall Control Strategy Based on Target Reaching for the Navigation of a Urban Electric Vehicle
} Jose Miguel Vilca Ventura, Lounis Adouane, Y. Mezouar, Pierre Lébraly

\section{To cite this version:}

Jose Miguel Vilca Ventura, Lounis Adouane, Y. Mezouar, Pierre Lébraly. An Overall Control Strategy Based on Target Reaching for the Navigation of a Urban Electric Vehicle. EEE/RSJ, IROS'13, International Conference on Intelligent Robots and Systems, Nov 2013, Tokyo, Japan. hal-01714846

\author{
HAL Id: hal-01714846 \\ https://hal.science/hal-01714846
}

Submitted on 23 Feb 2018

HAL is a multi-disciplinary open access archive for the deposit and dissemination of scientific research documents, whether they are published or not. The documents may come from teaching and research institutions in France or abroad, or from public or private research centers.
L'archive ouverte pluridisciplinaire HAL, est destinée au dépôt et à la diffusion de documents scientifiques de niveau recherche, publiés ou non, émanant des établissements d'enseignement et de recherche français ou étrangers, des laboratoires publics ou privés. 


\title{
An Overall Control Strategy Based on Target Reaching for the Navigation of an Urban Electric Vehicle*
}

\author{
José Vilca, Lounis Adouane, Youcef Mezouar and Pierre Lébraly
}

\begin{abstract}
This paper deals with reactive and flexible humanlike autonomous vehicle navigation. A human driver reactively guides his vehicle, performing a smooth trajectory within the roads limits until reaching the defined goal. To obtain a similar behavior with an unmanned ground vehicle (UGV), this paper proposes a flexible control law to drive a vehicle towards desired static or dynamic targets based on a novel definition of control variables and Lyapunov stability analysis. Moreover, a target assignment strategy, combined with an appropriate sigmoid function, that allow to perform smooth, flexible and safe vehicle navigation through successive waypoints is presented. The stability of the proposed control strategy is proved according to Lyapunov synthesis. Simulations and experiments are performed in different cases to demonstrate the reliability and efficiency of the control strategy.
\end{abstract}

\section{INTRODUCTION}

In the last decades, autonomous vehicles navigation have been a complex problem of major attention for the research community. Systems capable of performing efficient and robust autonomous navigation are useful in many robotic applications such as automatic industry, personal transportation, assistance to disabled or elder people, surveillance [1]. Even if many progress have been made, some specific technologies have to be improved for real application. In this paper, we are particularly interested in the field of the autonomous navigation of vehicles in an urban environment (cf. Fig. 1). This field allows to improve the transport systems for the reduction of traffic and pollution in large cities, among others. Different strategies in this field have been proposed in the literature [2], [3]. The most popular approaches are based on the following of a pre-defined reference trajectory. These approaches deal with three main groups: point stabilization (to reach a specific point with a certain orientation), trajectory tracking (to track a time parameterized references) and path following (to follow a path without explicit temporal references). For these approaches, the reference path can be defined by a combination of path planning and trajectory generation techniques [4].

Point stabilization for nonholonomic vehicle is a difficult problem of control system since the asymptotic stability of the system can not be guaranteed by a continuous statefeedback law [5]. Some works deal with this problem using the chained representation of the system [6] or a transformation in the robot kinematics and the Lyapunov

\footnotetext{
*This work was supported by the French National Research Agency (ANR) through the Safeplatoon and R-Discover projects

J. Vilca, L. Adouane, Y. Mezouar and P. Lébraly are with Institut Pascal, Blaise Pascal University - UMR CNRS 6602, Clermont-Ferrand, France FirstName. Lastname@univ-bpclermont.fr
}

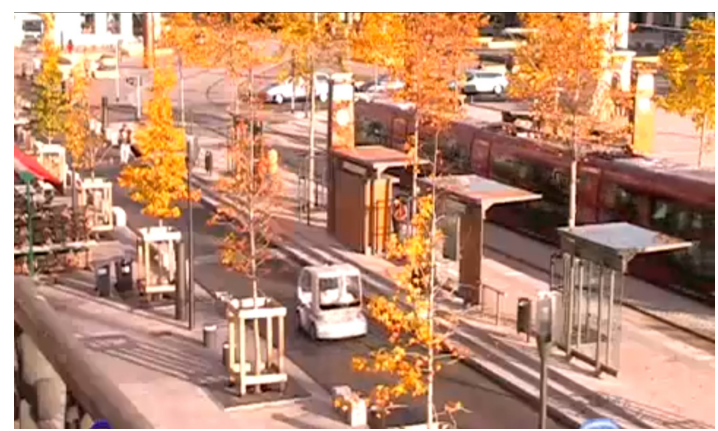

Fig. 1. Autonomous navigation of an electrical vehicle in an urban environment (Clermont-Ferrand, France).

stability method [7]. Nevertheless, the described approaches are applied only for point stabilization.

Different control methods for trajectory tracking and path following applied to wheeled mobile robots (unicycle, carlike robot, etc.) have been proposed in the literature [2], [8], [9] and [10]. In [9], [11] and [12], nonlinear control laws for trajectory tracking are synthesized for a unicycle robot using the Lyapunov stability analysis. The used Lyapunov functions are based only on the distance and orientation errors. A trajectory tracking control for a farm vehicle integrating sliding in the kinematic model is proposed in [8]. For path following problem, a control law for a tricycle robot is proposed in [10] and [13]. It is based on feedback linearization and system transformation to chained form representation [14]. Smoother convergence to desired path is achieved, in comparison with the performance obtained with trajectory tracking controllers. Nevertheless, the vehicle velocity tracks a desired velocity profile, while the path following controller acts only on the orientation to drive it towards the path.

Typically, to generate a reference path which must to be tracked by the vehicle, arc-lines, B-splines or a polynomial equation are used between points [15], [16] and [17]. In [3] a feasible path is obtained using a polynomial curvature spiral. In [18] straights line paths, defined by the position and orientation of a single waypoint, are considered. In this case, the orientation of the previous waypoint is not taken into account to simplify the implementation of the control law. This work proposes a navigation strategy which avoids the generation of a defined trajectory. The vehicle movements are obtained according to the proposed control law while considering sequential waypoints to reach and the vehicle constraints. The vehicle can perform different ma- 
neuvers between points without the necessity of replanning any reference trajectory. This strategy allows to perform a flexible navigation taking into account the waypoints suitably positioned in the environment.

In this work, a control law based on a novel definition of control variables and Lyapunov function is proposed. This function is based on the error distance, orientation and a new parameter related to the angle between the robot and the target position. The synthesized control law is deduced for point stabilization and trajectory tracking problem. Furthermore, a target assignment strategy is proposed to perform autonomous navigation through pre-defined waypoints. Consequently, the overall control strategy can also be exploited to achieve (static or dynamic) target-reaching.

The rest of the paper is organized as follows: the next section presents the problem description. Section III details the vehicle and target models, the proposed control law and its stability analysis. Section IV describes the target assignment strategy and the smooth switching between the targets. Simulation and experimental results are given in Section V. Finally, Section VI states the conclusion and future works.

\section{PROBLEM DESCRIPTION}

An important challenge in the field of autonomous vehicles consists of ensuring safe and flexible navigation in a structured environment (cf. Fig. 1 and 9). In this work, safe navigation consists in not colliding with the road limits and objects while respecting the vehicles physical constraints. Flexible navigation consists in allowing different maneuvers while guaranteeing a smooth trajectory. The considered scenario (cf. Fig. 9) is described below:

- The structured environment is a known road map where the roads have a specific width $w_{R}$.

- The vehicle model (kinematic) is supposed known.

- The vehicle starts at the initial position $P_{i}$ and it has to reach the final position $P_{f}$.

A safe reference path can be obtained by different algorithms such as Voronoï diagram [19]. In our case, this path is used to select defined points (waypoints). Therefore, the navigation problem is simplified to a waypoints tracking problem, i.e, the vehicle is guided by the waypoints (cf. Fig. 3) instead of following a specific fixed path. A single waypoint, defined by its position, orientation and velocity, is called a target. The control law to achieve the target is designed to generate a smooth and flexible vehicle navigation. Moreover, if the successive waypoints are close to each other then the vehicle performs a path following (cf. Section IV).

\section{TARGET-REACHING CONTROL}

The control strategy consists in driving an urban vehicle (cf. Fig. 1) towards specific target configuration (static or dynamic). The scenario is in an urban environment, thus the vehicle motion is low speed (less than $3 \mathrm{~m} / \mathrm{s}$ ) on asphalt road. Therefore, kinematic model of the vehicle relies on pure rolling, and dynamic effects and slippage are negligible.

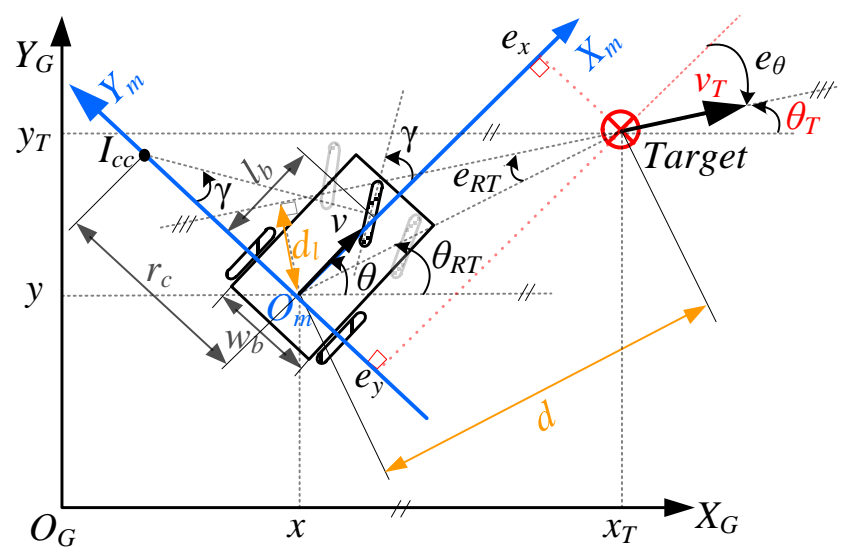

Fig. 2. Vehicle and target configuration in a Cartesian reference frame and the error variables.

This section gives the details of the vehicle and target statespace model, the proposed control law and the proof of its stability. Before to start, let us describe the notation illustrated in Fig. 2 below:

- $O_{G}$ and $O_{m}$ are respectively the origin of global and local reference frames.

- $(x, y, \theta)$ and $\left(x_{T}, y_{T}, \theta_{T}\right)$ are respectively the tricycle and target postures in the global reference frame.

- $v$ and $v_{T}$ are respectively the linear velocity of the tricycle and target.

- $\gamma$ is the orientation of the vehicle front wheel.

- $I_{c c}$ is the instantaneous center of curvature of the vehicle trajectory with $c_{c}=1 / r_{c}$ as the curvature and the radius of curvature is $r_{c}=l_{b} / \tan (\gamma)$.

- $l_{b}$ and $w_{b}$ are respectively the wheelbase and the track width of the vehicle.

- $\left(e_{x}, e_{y}, e_{\theta}\right)$ are the errors w.r.t local frame $\left(X_{m} Y_{m}\right)$ between the tricycle and target postures.

- $\theta_{R T}$ and $d$ are respectively the angle and distance between the target and vehicle positions.

- $e_{R T}$ is the error related to the vehicle position $(x, y)$ w.r.t the target orientation.

- $d_{l}$ is the distance from the vehicle to the target orientation line.

\section{A. Vehicle and target modeling}

The kinematic model of the urban vehicle is based on the well-known tricycle model [6]. The two front wheels are replaced by a single virtual wheel located at the center between the front wheels. It is given below (cf. Fig. 2)

$$
\left\{\begin{array}{l}
\dot{x}=v \cos (\theta) \\
\dot{y}=v \sin (\theta) \\
\dot{\theta}=v \tan (\gamma) / l_{b}
\end{array}\right.
$$

To simplify the controller computation and to use the general model of robot motion, let us consider a dynamic target as a point with nonholonomic constraints (cf. Fig. 2). 
Its kinematic is given by:

$$
\left\{\begin{array}{l}
\dot{x}_{T}=v_{T} \cos \left(\theta_{T}\right) \\
\dot{y}_{T}=v_{T} \sin \left(\theta_{T}\right) \\
\dot{\theta}_{T}=\omega_{T}
\end{array}\right.
$$

where $\omega_{T}$ is the angular velocity of the target. The radius of curvature is computed by $r_{c_{T}}=v_{T} / \omega_{T}$. An important consideration for target-reaching is that $v_{T} \leq v_{\max }$ and $r_{c_{T}} \geq r_{c_{\min }}$, where $v_{\max }$ and $r_{c_{\min }}$ are respectively the maximum linear velocity and the minimum radius of curvature of the vehicle. For the static target-reaching, $\omega_{T}$ is considered equal to zero and $v_{T}$ is a desired velocity value for the vehicle when it reaches the desired posture $\left(x_{T}, y_{T}, \theta_{T}\right)$.

\section{B. Control Law}

Before to present the proposed control law, let us introduce the error variables. The errors $\left(e_{x}, e_{y}, e_{\theta}\right)$ are given by:

$$
\begin{cases}e_{x}=\cos (\theta)\left(x_{T}-x\right) & +\sin (\theta)\left(y_{T}-y\right) \\ e_{y}=-\sin (\theta)\left(x_{T}-x\right) & +\cos (\theta)\left(y_{T}-y\right) \\ e_{\theta}=\theta_{T}-\theta & \end{cases}
$$

In this paper, a new error function $e_{R T}$ is added to the canonical error system (3) (cf. Fig. 2). Let us first give the expression of $d$ and $\theta_{R T}$ as (cf. Fig. 2):

$$
\begin{aligned}
& d=\sqrt{\left(x_{T}-x\right)^{2}+\left(y_{T}-y\right)^{2}} \\
& \begin{cases}\theta_{R T}=\arctan \left(\left(y_{T}-y\right) /\left(x_{T}-x\right)\right) & \text { if } d>\xi \\
\theta_{R T}=\theta_{T} & \text { if } d \leq \xi\end{cases}
\end{aligned}
$$

where $\xi$ is a small positive value $(\xi \approx 0)$. The error $e_{R T}$ is defined as (cf. Fig. 2):

$$
e_{R T}=\theta_{T}-\theta_{R T}
$$

It can be written as a function of $e_{x}, e_{y}$ and $e_{\theta}$ as:

$$
\begin{aligned}
\tan \left(e_{R T}\right)= & \tan \left(e_{\theta}-\left(\theta_{R T}-\theta\right)\right) \\
& =\frac{\tan \left(e_{\theta}\right)-\tan \left(\theta_{R T}-\theta\right)}{1+\tan \left(e_{\theta}\right) \tan \left(\theta_{R T}-\theta\right)} \\
& =\frac{e_{x} \tan \left(e_{\theta}\right)-e_{y}}{e_{x}+\tan \left(e_{\theta}\right) e_{y}}
\end{aligned}
$$

where $\tan \left(\theta_{R T}-\theta\right)=e_{y} / e_{x}$ (cf. Fig. 2).

The derivatives of the errors (3) and (6) can be obtained using (1), (2), (5) and (4):

$$
\begin{aligned}
\dot{e}_{x}= & \cos (\theta)\left(\dot{x}_{T}-\dot{x}\right)+\sin (\theta)\left(\dot{y}_{T}-\dot{y}\right) \\
& -\sin (\theta)\left(x_{T}-x\right) \dot{\theta}+\cos (\theta)\left(y_{T}-y\right) \dot{\theta} \\
= & -v+e_{y} \dot{\theta}+v_{T} \cos \left(\theta_{T}\right) \cos (\theta)+v_{T} \sin \left(\theta_{T}\right) \sin (\theta) \\
= & -v+e_{y} v \tan (\gamma) / l_{b}+v_{T} \cos \left(e_{\theta}\right) \\
\dot{e}_{y}= & -\sin (\theta)\left(\dot{x}_{T}-\dot{x}\right)+\cos (\theta)\left(\dot{y}_{T}-\dot{y}\right) \\
& -\cos (\theta)\left(x_{T}-x\right) \dot{\theta}-\sin (\theta)\left(y_{T}-y\right) \dot{\theta} \\
= & -e_{x} \dot{\theta}-v_{T} \cos \left(\theta_{T}\right) \sin (\theta)+v_{T} \sin \left(\theta_{T}\right) \cos (\theta) \\
= & -e_{x} v \tan (\gamma) / l_{b}+v_{T} \sin \left(e_{\theta}\right) \\
\dot{e}_{\theta}= & \dot{\theta}_{T}-\dot{\theta} \\
= & \frac{v_{T}}{r_{c_{T}}}-v \tan (\gamma) / l_{b}
\end{aligned}
$$

$$
\begin{aligned}
\dot{e}_{R T}= & \dot{\theta}_{T}-\dot{\theta}_{R T} \\
= & \frac{v_{T}}{r_{c_{T}}}-\frac{v_{T} \sin \left(\theta_{T}\right)\left(x_{T}-x\right)-v_{T} \cos \left(\theta_{T}\right)\left(y_{T}-y\right)}{d^{2}} \\
& -\frac{-v \sin (\theta)\left(x_{T}-x\right)+v \cos (\theta)\left(y_{T}-y\right)}{d^{2}} \\
= & \frac{v_{T}}{r_{c_{T}}}-\frac{v_{T} e_{x} \sin \left(e_{\theta}\right)}{d^{2}}+\frac{v_{T} e_{y} \cos \left(e_{\theta}\right)}{d^{2}}-\frac{e_{y} v}{d^{2}}
\end{aligned}
$$

The control law to reach a target (static or dynamic) is obtained using Lyapunov stability analysis framework. The desired linear velocity $v$ and the front wheel orientation $\gamma$ of the vehicle that make the errors $\left(e_{x}, e_{y}, e_{\theta}\right)$ converge to zero can be chosen as:

$$
\begin{aligned}
& v=v_{T} \cos \left(e_{\theta}\right)+v_{b} \\
& \gamma=\arctan \left(l_{b} c_{c}\right)
\end{aligned}
$$

where $v_{b}$ and $c_{c}$ are given by:

$$
v_{b}=K_{x}\left(K_{d} e_{x}+K_{l} d \sin \left(e_{R T}\right) \sin \left(e_{\theta}\right)+K_{o} \sin \left(e_{\theta}\right) c_{c}\right)
$$

$$
\begin{aligned}
c_{c}= & \frac{1}{r_{c_{T}} \cos \left(e_{\theta}\right)}+\frac{d^{2} K_{l} \sin \left(e_{R T}\right) \cos \left(e_{R T}\right)}{r_{c_{T}} K_{o} \sin \left(e_{\theta}\right) \cos \left(e_{\theta}\right)}+K_{\theta} \tan \left(e_{\theta}\right) \\
& +\frac{K_{d} e_{y}-K_{l} d \sin \left(e_{R T}\right) \cos \left(e_{\theta}\right)}{K_{o} \cos \left(e_{\theta}\right)}+\frac{K_{R T} \sin ^{2}\left(e_{R T}\right)}{\sin \left(e_{\theta}\right) \cos \left(e_{\theta}\right)}
\end{aligned}
$$

where $\mathbf{K}=\left(K_{d}, K_{l}, K_{o}, K_{x}, K_{R T}, K_{\theta}\right)$ is a vector of positive constants which must be defined by the designer. These constants can depend on the initial configuration such as initial distance (reference values are given in Section V) to adapt the performance of controller for different initial configurations. We observed that $v_{b}$ is a function of posture error and the curvature command $c_{c}$. It allows to manage the linear velocity when the vehicle has a great curvature according to $e_{\theta}$. Moreover, $c_{c}$ depends mainly on the error posture.

\section{Stability analysis}

This stability analysis based on Lyapunov method is used to determine the convergence of the vehicle to the target posture, i.e., for a finite time, the error system $\left(e_{x}, e_{y}, e_{\theta}\right)$ converges to zero [20]. Let us first define the Lyapunov function $V$ by (16). It is a function of three parameters which depend on: the distance $d$ between the target and vehicle positions, the distance $d_{l}$ from the vehicle to the target line (line that pass through the target position with orientation equal to the target orientation), this term is related to the Line of Sight and Flight of the target [21], and the orientation error $e_{\theta}$ between the vehicle and the target (cf. Fig. 2). It is represented by:

$$
\begin{aligned}
V & =\frac{1}{2} K_{d} d^{2}+\frac{1}{2} K_{l} d_{l}^{2}+K_{o}\left[1-\cos \left(e_{\theta}\right)\right] \\
& =\frac{1}{2} K_{d} d^{2}+\frac{1}{2} K_{l} d^{2} \sin ^{2}\left(e_{R T}\right)+K_{o}\left[1-\cos \left(e_{\theta}\right)\right]
\end{aligned}
$$

where the initial values of $e_{R T}$ and $e_{\theta}$ satisfy:

$$
\left.e_{R T} \in\right]-\pi / 2, \pi / 2\left[\text { and } e_{\theta} \in\right]-\pi / 2, \pi / 2[
$$


These conditions (17) guarantee that the target is ahead to the vehicle w.r.t. its orientation. Moreover, (17) has open interval that allows to avoid local minimum. Therefore, $V$ is a positive-definite function [20].

The Lyapunov function (16) can be written according to $e_{x}, e_{y}$ as follows:

$$
V=\frac{1}{2}\left(e_{x}^{2}+e_{y}^{2}\right)\left[K_{d}+K_{l} \sin ^{2}\left(e_{R T}\right)\right]+K_{o}\left[1-\cos \left(e_{\theta}\right)\right]
$$

To guarantee the system stability, $\dot{V}$ has to be negativedefinite [20]. By taking the derivate of (18) and using (8), (9), (10), (11), (12) and (13), $\dot{V}$ can be written:

$$
\begin{aligned}
\dot{V}= & \left(e_{x} \dot{e}_{x}+e_{y} \dot{e}_{y}\right)\left[K_{d}+K_{l} \sin ^{2}\left(e_{R T}\right)\right] \\
& +K_{l} d^{2} \sin \left(e_{R T}\right) \cos \left(e_{R T}\right) \dot{e}_{R T}+K_{o} \sin \left(e_{\theta}\right) \dot{e}_{\theta} \\
= & {\left[-e_{x} v_{b}+v_{T} e_{y} \sin \left(e_{\theta}\right)\right]\left[K_{d}+K_{l} \sin ^{2}\left(e_{R T}\right)\right] } \\
+ & K_{l} \sin \left(e_{R T}\right) \cos \left(e_{R T}\right)\left[\frac{d^{2} v_{T}}{r_{c_{T}}}-v_{T} e_{x} \sin \left(e_{\theta}\right)-e_{y} v_{b}\right] \\
+ & K_{o} \sin \left(e_{\theta}\right)\left(\frac{v_{T}}{r_{c_{T}}}-v_{T} \cos \left(e_{\theta}\right) c_{c}-v_{b} c_{c}\right)
\end{aligned}
$$

Using (7) in the first two terms of (19) and factorizing the common terms, it holds that:

$$
\begin{aligned}
\dot{V}= & v_{T} \sin \left(e_{\theta}\right)\left[K_{d} e_{y}-K_{l} d \sin \left(e_{R T}\right) \cos \left(e_{\theta}\right)\right] \\
& +\frac{v_{T}}{r_{c_{T}}}\left[d^{2} K_{l} \sin \left(e_{R T}\right) \cos \left(e_{R T}\right)+K_{o} \sin \left(e_{\theta}\right)\right] \\
& -v_{b}\left[K_{d} e_{x}+K_{l} d \sin \left(e_{R T}\right) \sin \left(e_{\theta}\right)+K_{o} \sin \left(e_{\theta}\right) c_{c}\right] \\
& -v_{T} K_{o} \sin \left(e_{\theta}\right) \cos \left(e_{\theta}\right) c_{c}
\end{aligned}
$$

Finally, using (14) and (15) in (20), we obtain:

$$
\begin{aligned}
\dot{V}= & -K_{x}\left[K_{d} e_{x}+K_{l} d \sin \left(e_{R T}\right) \sin \left(e_{\theta}\right)+K_{o} \sin \left(e_{\theta}\right) c_{c}\right]^{2} \\
& -v_{T} K_{o} K_{\theta} \sin ^{2}\left(e_{\theta}\right)-v_{T} K_{o} K_{R T} \sin ^{2}\left(e_{R T}\right) \leq 0
\end{aligned}
$$

Eq. (21) shows that the system is stable while the initial conditions (17) are satisfied. To ensure the asymptotic stability of the error system, $\dot{V}$ has to be a negative-definite function. Let us exhibit the case where $\dot{V}=0$ with $v_{T}>0$ and $v_{T}=0$. Firstly, when $v_{T}>0$ and using the initial assumption $\mathbf{K}>0$, it is straightforward to show that $e_{x}, e_{\theta}, e_{R T}$ are equal to zero to satisfy (21), then according to (6), (5) and (17) $d$ is equal to zero $\left(e_{y}=0\right)$. Hence, $\dot{V}$ is equal to zero when $v_{T}>0$, only if $\left(e_{x}, e_{y}, e_{\theta}\right)=(0,0,0)$.

Secondly, let us consider the case where $v_{T}=0$. The initial assumption is identical. Hence, the second and third terms of (21) are equal to zero when $v_{T}=0$. Additionally, when $v_{T}=0$, we consider that $r_{c_{T}} \rightarrow \infty$, consequently the first term of $\dot{V}$ is equal to zero when:

$$
K_{d} e_{x}+K_{l} d \sin \left(e_{R T}\right) \sin \left(e_{\theta}\right)+K_{o} \sin \left(e_{\theta}\right) c_{c}=0
$$

Replacing (15) with $r_{c_{T}} \rightarrow \infty$ in (22), the following expression is obtained:

$$
\begin{aligned}
0= & K_{d} e_{x}+K_{l} d \sin \left(e_{R T}\right) \sin \left(e_{\theta}\right) \\
& +\tan \left(e_{\theta}\right)\left[K_{d} e_{y}-K_{l} d \sin \left(e_{R T}\right) \cos \left(e_{\theta}\right)\right] \\
& +K_{o} \sin \left(e_{\theta}\right)\left[K_{\theta} \tan \left(e_{\theta}\right)+\frac{K_{R T} \sin ^{2}\left(e_{R T}\right)}{\sin \left(e_{\theta}\right) \cos \left(e_{\theta}\right)}\right] \\
= & K_{d}\left[e_{x}+e_{y} \tan \left(e_{\theta}\right)\right]+K_{o} K_{\theta} \frac{\sin ^{2}\left(e_{\theta}\right)}{\cos \left(e_{\theta}\right)} \\
& +K_{o} K_{R T} \frac{\sin ^{2}\left(e_{R T}\right)}{\cos \left(e_{\theta}\right)}
\end{aligned}
$$

Using (7) in (23), we obtain:

$K_{d} d \frac{\cos \left(e_{R T}\right)}{\cos \left(e_{\theta}\right)}+K_{o} K_{\theta} \frac{\sin ^{2}\left(e_{\theta}\right)}{\cos \left(e_{\theta}\right)}+K_{o} K_{R T} \frac{\sin ^{2}\left(e_{R T}\right)}{\cos \left(e_{\theta}\right)}=0$

Eq. (24) exhibits quadratic terms. Consequently, considering the initial conditions (17), $\cos \left(e_{R T}\right)$ and $\cos \left(e_{\theta}\right)>0$ are greater than zero. Therefore, all the terms of (24) are positive and they must be equal to zero, i.e., $d, e_{\theta}, e_{R T}=0$, and if $d=0$ then $e_{x}, e_{y}=0$. Hence, from (24), $\dot{V}$ is equal to zero when $v_{T}=0$ and $r_{c_{T}} \rightarrow \infty$, only if $\left(e_{x}, e_{y}, e_{\theta}\right)=(0,0,0)$.

Conclusively, if $v_{T}>0$ and $v_{T}=0, V$ is always strictly positive and $\dot{V}$ is always strictly negative while $\left(e_{x}, e_{y}, e_{\theta}\right) \neq$ $(0,0,0)$. Therefore, the errors system is asymptotically stable while the initial vehicle conditions (17) are satisfied.

\section{SMOOTH NAVIGATION THROUGH SEQUENTIAL TARGETS}

This section shows the strategy to navigate through successive waypoints (targets) and the method applied on the controller variables to guarantee smooth navigation between the targets, specifically when the switches occur.

\section{A. Sequential target assignment}

The proposed strategy uses a sequence of sorted waypoints suitably located in the environment. For safe navigation, the line that joins two successive waypoints is in the freecollision area (cf. Fig. 3). The method to select the optimal number of waypoints (target set-point $\left(x_{T_{j}}, y_{T_{j}}, \theta_{T_{j}}, v_{T_{j}}\right)$ ) in order to perform safe vehicle navigation will be addressed in future works. In this paper, we consider for simplicity that the waypoints are uniformly positioned in the skeleton of the Voronoï diagram of the known environment. Safe navigation between waypoints is guaranteed by imposing $E_{d i s t}$ and $E_{\text {angle }}$ (cf. Fig. 3) which allow to guide the vehicle to reach the assigned current target $T_{j}$ with an appropriate conditions to reach the next target $T_{j+1}$.

The strategy to assign the next target is shown in Algorithm 1. The parameters of the control law allow to the vehicle to reach each target (cf. Section V) while ensuring that the vehicle trajectory is always inside the road limits (cf. Fig. 3).

The current target $T_{j}=\left(x_{T_{j}}, y_{T_{j}}\right)$ is defined by the following parameters: $D_{j}$ the distance between the last target $T_{j-1}=\left(x_{T_{j-1}}, y_{T_{j-1}}\right)$ and the current target $T_{j} ; \theta_{T_{j}}$ the 


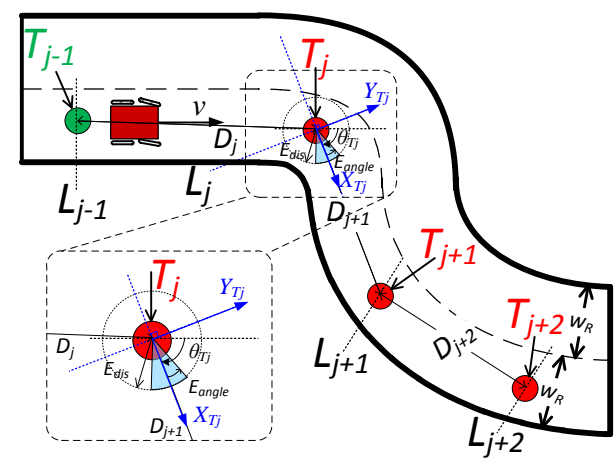

Fig. 3. Description of waypoints and the target assignment

Input : Vehicle posture, current target $T_{j}$ and a set of $N$ waypoints

Output: Switch between target set-points

if $\left(d \leq E_{d i s}\right.$ and $\left.e_{\theta} \leq E_{\text {angle }}\right)$ or $\left(x^{T_{j}} \geq 0\right)$

$\left\{x^{T_{j}}\right.$ is the coordinate of the vehicle w.r.t current

Target frame $X_{T_{j}} Y_{T_{j}}$ (cf. Fig. 3) $\}$ then

Switch between the current target $T_{j}$ and the next waypoints $T_{j+1}$;

else

Go to the current target $T_{j}$;

end

Algorithm 1: Sequential target assignment

orientation between $T_{j}$ and $T_{j+1}=\left(x_{T_{j+1}}, y_{T_{j+1}}\right)$ given by:

$$
\theta_{T_{j}}=\arctan \left(\left(y_{T_{j+1}}-y_{T_{j}}\right) /\left(x_{T_{j+1}}-x_{T_{j}}\right)\right)
$$

\section{B. Smooth switching between targets}

The idea here is to obtain a smooth evolution of the errors avoiding discontinuities in the control law (which could induce jerking in the vehicle movement). Indeed, when the vehicle switches from target e.g., $T_{j}$ to $T_{j+1}$, the value of controller variables $\mathbf{C}_{v}=\left(e_{x}, e_{y}, e_{\theta}, e_{R T}, v_{T}, r_{c_{T}}\right)$ can change abruptly (cf. Subsection V-A). In this work, we propose to avoid this hard switch by introducing a virtual evolution of this control variables for a certain amount of movement distance $d_{s}$ (smoothness distance) without disturbing the safe navigation. This distance depends on initial distance $\left(\%\right.$ of $d_{i}$ ) separating the robot and $T_{j+1}$.

A Sigmoid function is applied to the controller variables $\mathbf{C}_{v}$ along of $d_{s}$. The new Smooth Virtual Controller variables $\left(\mathbf{S V C}_{v}\right)$ are designed according to the covered distance $d_{c}=$ $d_{i}-d$, where $d$ is the current distance to the current target (cf. Fig. 4). The $\mathbf{S V C}_{v}$ function is given by:

$$
\mathbf{S V C}_{v}\left(d_{c}\right)=\mathbf{C}_{v i}+\frac{\left(\mathbf{C}_{v}-\mathbf{C}_{v i}\right)}{1+e^{-a\left(d_{c}-d_{0}\right)}}
$$

where $\mathbf{C}_{v i}$ and $\mathbf{C}_{v}$ are respectively the initial and current values of the controller variables. For example, for $e_{x}$, an element of $\mathbf{C}_{v}$, when the target switches from $T_{j}$ to $T_{j+1}$, $e_{x i}$ is the value before to switch to $T_{j+1}$ and $e_{x}$ is the current error w.r.t. the target $T_{j+1} ; d_{0}$ is the value where the function

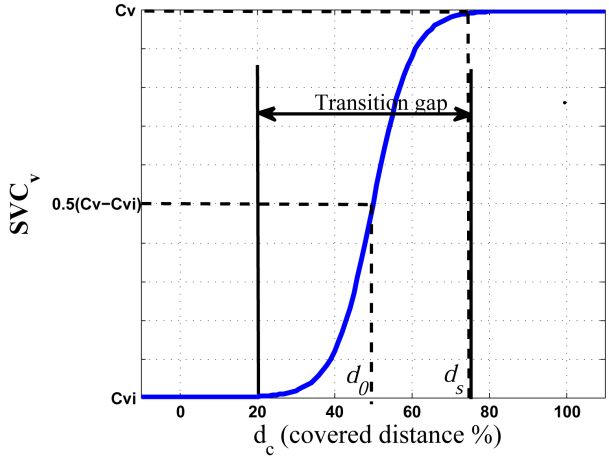

Fig. 4. Evolution of the $\mathbf{S V C}_{v}$ used to ensure smooth control when the target switching occurs.

has a half of its current value and $a$ is a constant value related to the slope of the sigmoid function. It is designed to attain the effective value $\left(\mathbf{S V C} v \approx \mathbf{C}_{v}\right.$ ) when $d_{c}=d_{s}$ (cf. Fig. 4).

\section{VALIDATION}

This section presents simulations and experiments to demonstrate the efficiency of the control law for targetreaching (static and dynamic) and autonomous navigation in structured environment. The physical parameters of the urban vehicle VIPALAB, modeled using the tricycle kinematics, were considered (cf. Section III).

\section{A. Simulation results}

This section shows, in simulation, the performance (safety, smoothness and convergence) of the control law to reach a desired target (static or dynamic) located at different distances. For each simulation the vehicle starts at the same configuration but with different values of target velocities.

Fig. 5 shows the trajectories of the vehicle for different static targets $\left(T_{i}, i=1, \ldots, 6\right)$ and for a dynamic target $T_{d}$ (sinusoidal trajectory). The static target are positioned at different initial distances $d_{i}$ and orientation angle between them ( $45^{\circ}$ until $T_{5}$ and $0^{\circ}$ for $T_{6}$ ). The velocity profile of the targets for each simulation are $v_{T}=0.1,0.5$ and $1.0 \mathrm{~m} / \mathrm{s}$ respectively. The values of the controller parameters are $\mathbf{K}=\left(1 / d_{i}, 1.8,8,0.15,0.6,0.01\right)\left(d_{i}\right.$ is the initial distance

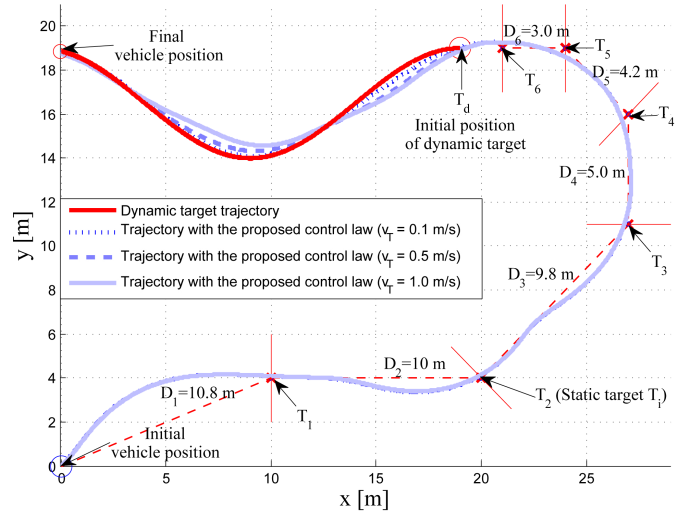

Fig. 5. Trajectories of the vehicle for several target velocities. 

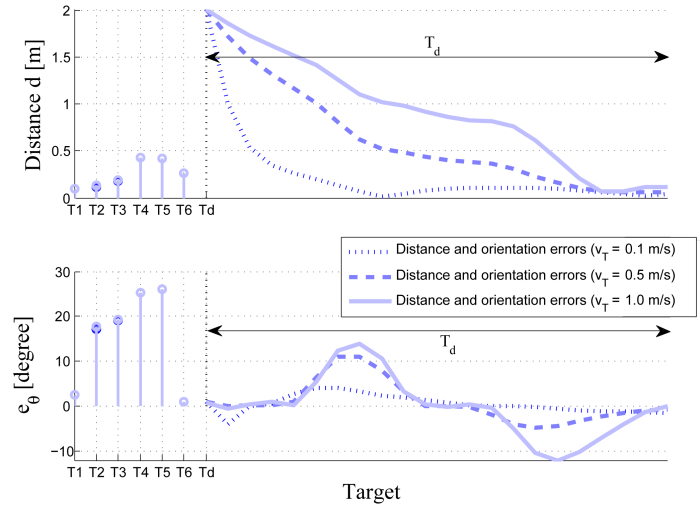

Fig. 6. Distance and orientation errors of the vehicle for several target velocities.
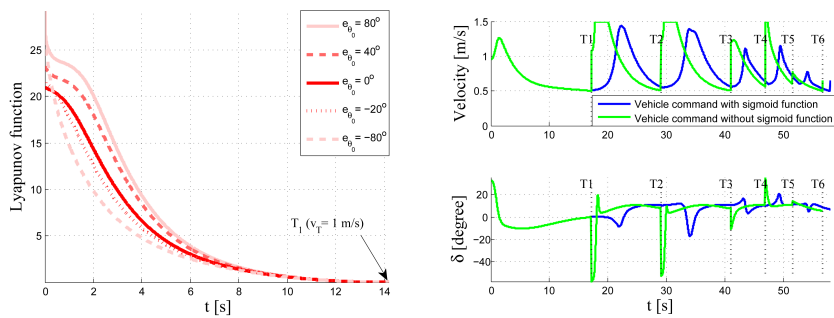

Fig. 7. Values of the Lyapunov function for several initial orientations to reach $T_{1}$.

Fig. 8. Control commands with and without adaptive Sigmoid $\left(\mathbf{S V C}_{v}\right)$ use.

to the target). These parameters were chosen to obtain a safe and smooth trajectory, fast response and velocity value within the limit of the vehicle, which are $v_{\max }=1.5 \mathrm{~m} / \mathrm{s}$ and $r_{c_{\min }}=3.8 \mathrm{~m}\left(\gamma_{\max }= \pm 19^{\circ}\right)$. It is noted that the vehicle converges to each target (static and dynamic), located in different positions and with different set of velocities. The dynamic target starts its movement when the vehicle reaches the last static target $T_{6}$.

Fig. 6 shows the values of errors $d$ and $e_{\theta}$ for the different targets to reach. For static targets $\left(T_{i}, i=1, \ldots, 6\right)$, the obtained values of errors just before to switch from target $T_{i}$ to $T_{i+1}$ are shown. For dynamic target, the evolution of $d(t)$ and $e_{\theta}(t)$ during all tracking phase are shown. It is observed that the distance and orientation errors of static targets depend on initial configuration (distance and orientation). The errors increase when the static targets are closer. Moreover, for static targets where the profile velocity is the desired vehicle velocity at target position, the different profiles velocities have similar errors. It occurs in static target because the proposed control law (angle steering) relies only on the dynamic of posture errors between the vehicle and the static target (cf. eqs. (13) and (15)) (the target velocity is a set-point for the vehicle when it reaches the target posture (cf. eq. (12)) and the controller parameters $\mathbf{K}$ are tunning according to same initial distance $d_{i}$ to the target. Obviously, for dynamic target, the small target profile velocity has a faster convergence to zero.

The convergence of the Lyapunov function (16) is shown in Fig. 7 (cf. Subsection III-C) when the vehicle starts at the same initial position but with different initial orientations to the target $T_{1}$ and while taking $v_{T}=1.0 \mathrm{~m} / \mathrm{s}$.

The use of Sigmoid function is observed in the vehicle commands (velocity and steering angle) (cf. Fig. 8) for the static target with profile velocity of $v_{T}=0.5$. It is noted that the Sigmoid function contributes to avoid peaks at the transition time and to obtain thus smooth vehicle commands while maintaining the stability of the control.

\section{B. Experimental results}

The experiment was made to show the performance of the control law and target assigment strategy using the waypoints sampling of the defined reference trajectory at each $1 \mathrm{~m}$. This selected distance guarantees that the line between two successive waypoints does not collide with the road limits. Hence, the controller parameters $\mathbf{K}$ are constants.

Our strategy was implemented in the VIPALAB urban vehicle (cf. Fig. 9(a)). The dimensions of the vehicle are $1.27 \mathrm{~m}$ (width), $1.96 \mathrm{~m}$ (length) and $2.11 \mathrm{~m}$ (height). This vehicle is embedded with different sensors to obtain information about the environment and the vehicle such as

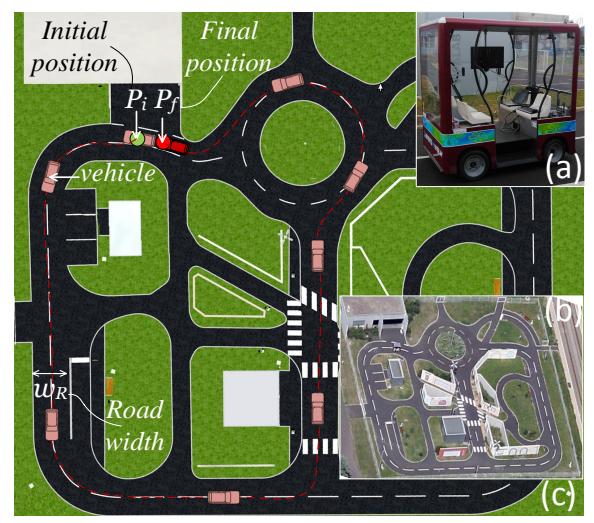

Fig. 9. (a) VIPALAB electric urban vehicle, (b) PAVIN experimental platform, (c) Top view of the virtual PAVIN.

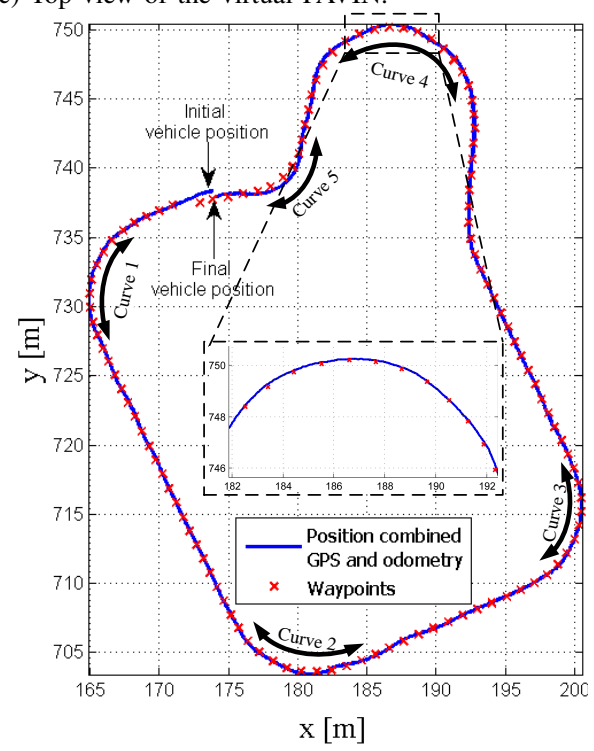

Fig. 10. GPS real Trajectory of the vehicle and waypoints. 


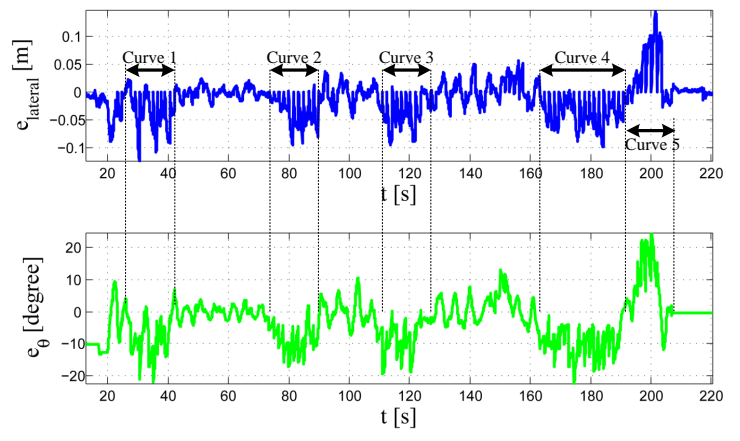

Fig. 11. Lateral distance and orientation errors of the vehicle w.r.t. the line which link the current target and the last one (real experiment).
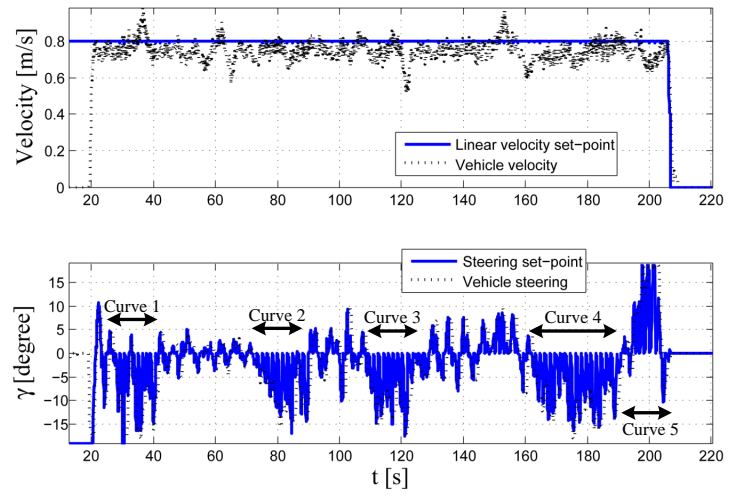

Fig. 12. Control commands of the vehicle (real experiment).

odometers, steering angle sensor and GPS-RTK (its accuracy is $0.02 \mathrm{~m}$ ). They are used to estimate the vehicle position and orientation. The used sample time is $0.01 s$. Navigation was performed in a structured environment named PAVIN (Plateforme d'Auvergne pour Véhicules INtelligents) (cf. Fig 9 (b)).

It can be seen in Fig. 10 that the vehicle follows the waypoints successfully. Moreover, the trajectory using the proposed strategy is close to the reference path (Fig. 10 and 11). Fig. 11 shows that the errors w.r.t. reference trajectory are greater when the vehicle moves to a curve in the path. Fig. 12 shows the commands sent to the vehicle and the real values taken from the vehicle of the velocity and steering angle. It is noted that the real values have noise signals that are related to the sensor inaccuracies. The video enclosed to this paper shows the performance of this experiment.

\section{CONCLUSION}

This paper presented an overall control strategy to cope with the problem of navigation in a structured environment. A control law was synthesized using a suitable Lyapunov function, which takes into account the position, angle between the robot and the target and orientation such as setpoints. Moreover, it allows to perform the target-reaching (static and dynamic). The stability of the overall control architecture was proved using a suitable Lyapunov function based on the new set of variables. A target assignment strategy to perform a safe navigation was also presented. It is based on the target switching using waypoints and sigmoid function that allow to obtain a smooth, flexible and safe vehicle trajectory. Simulations and experiments using real urban vehicle show the efficiency and the flexibility of the proposed control strategy for the navigation of an urban vehicle.

In future works, we will show the advantages of the proposed overall control strategy in comparison to other approaches. The optimal selection of waypoints (minimum number of waypoints) in a reference path to perform safer and smoother trajectory will be proposed. The error limits $E_{d i s}$ and $E_{\text {angle }}$ will consider the inaccuracies of the vehicle sensors. Moreover, this strategy will be applied to multi-robot system for a virtual structure approach.

\section{REFERENCES}

[1] B. Siciliano and O. Khatib, Eds., Springer Handbook of Robotics, Part F. Springer, 2008.

[2] L. Adouane, A. Benzerrouk, and P. Martinet, "Mobile robot navigation in cluttered environment using reactive elliptic trajectories," in 18th IFAC World Congress, August 2011.

[3] T. Gu and J. M. Dolan, "On-road motion planning for autonomous vehicles," in Intelligent Robotics and Applications, C.-Y. Su, S. Rakheja, and H. Liu, Eds. Springer Berlin Heidelberg, 2012, vol. 7508.

[4] S. M. LaValle, Planning Algorithms. Cambridge Univ. Press, 2006.

[5] R. W. Brockett, "Asymptotic stability and feedback stabilization," Differential Geometric Control Theory, pp. 181 - 191, 1983.

[6] A. D. Luca, G. Oriolo, and C. Samson, "Feedback control of a nonholonomic car-like robot," in Robot Motion Planning and Control, J.-P. Laumond, Ed. Berlin: Springer-Verlag, 1998, pp. 171-253.

[7] F. Pourboghrat, "Exponential stabilization of nonholonomic mobile robots," Computers \& Electrical Engineering, vol. 28, no. 5, 2002.

[8] H. Fang, R. Lenain, B. Thuilot, and P. Martinet, "Trajectory tracking control of farm vehicles in presence of sliding," in IEEE/RSJ International Conference on Intelligent Robots and Systems, 2005, pp. 58-63.

[9] S. Blazic, "Four-state trajectory-tracking control law for wheeled mobile robots," in 10th International IFAC Symposium on Robot Control. Croatia, September 2012.

[10] A. V. Pesterev, "Stabilizing control for a wheeled robot following a curvilinear path," in 10th International IFAC Symposium on Robot Control. Croatia, September 2012.

[11] J. Vilca, L. Adouane, and Y. Mezouar, "Reactive navigation of a mobile robot using elliptic trajectories and effective online obstacle detection," Gyroscopy and Navigation, vol. 4, pp. 14 - 25, 2013.

[12] Y. Kanayama, Y. Kimura, F. Miyazaki, and T. Noguchi, "A stable tracking control method for an autonomous mobile robot," in Proceedings of the IEEE International Conference on Robotics and Automation, May 1990, pp. 384 - 389.

[13] C. Samson, "Control of chained systems. application to path following and time-varying point-stabilization of mobile robots," IEEE Transactions on Automatic Control, vol. 40, no. 1, pp. 64-77, January 1995.

[14] J.-P. Laumond, La Robotique Mobile, ser. Traité IC2 InformationCommande-Communication. Hermès, 2001.

[15] J. Horst and A. Barbera, "Trajectory generation for an on-road autonomous vehicle," Proceedings of the SPIE: Unmanned Systems Technology VIII, 2006.

[16] J. Connors and G. H. Elkaim, "Manipulating b-spline based paths for obstacle avoidance in autonomous ground vehicles," in ION National Technical Meeting, ION NTM 2007, San Diego, CA, USA, 2007.

[17] J.-W. Lee and B. Litkouhi, "A unified framework of the automated lane centering/changing control for motion smoothness adaptation," in 15th International IEEE Conference on Intelligent Transportation Systems (ITSC), 2012, pp. 282-287.

[18] J. Courbon, Y. Mezouar, and P. Martinet, "Autonomous navigation of vehicles from a visual memory using a generic camera model," Intelligent Transport System (ITS), vol. 10, pp. 392-402, 2009.

[19] J.-C. Latombe, Robot Motion Planning. Kluwer Academic Publishers, Boston, MA, 1991.

[20] H. K. Khalil, Nonlinear Systems, 3rd ed. Prentice Hall, 2002.

[21] G. M. Siouris, Missile Guidance and Control Systems. SpringerVerlag, 2004. 